\title{
Mortality in patients with epilepsy: a study of patients in long term residential care
}

\author{
P Klenerman, J W A S Sander, S D Shorvon
}

\begin{abstract}
The causes of death in a group of patients with severe epilepsy in long term residential care over a period of 11 years were assessed and the standardised mortality rate (SMR) determined. A total of 3392 patient-years were surveyed. One hundred and thirteen deaths were recorded in the period and this represents an overall mortality rate which is almost twice the expected rate for this population (SMR = $1 \cdot 9$; 95\% CI 1.6-2.3; p < 0.01). Most deaths were due to cancer $(26 \%)$, bronchopneumonia $(25 \%)$, circulatory diseases $(24 \%)$, were seizure-related $(12 \%)$ or due to sudden unexpected death $(6 \%)$. The highest SMRs in the neoplasm sub-group were due to cancers of the pancreas $(S M R=$ 6.2) and hepatobiliary tumours (SMR = 17.6). Twenty per cent of patients died of epilepsy or epilepsy related causes (that is accidents, during seizures, status or sudden unexpected death). One in every 480 patients died due to a sudden unexpected death. This study in a highly selected population seems to confirm suggestions that mortality rates are higher in patients with epilepsy than in the general population, but prospective studies are warranted to ascertain underlying mechanisms.
\end{abstract}

\section{(F Neurol Neurosurg Psychiatry 1993;56:149-152)}

It has been suggested that patients with epilepsy have an increased mortality rate-approximately two to three times that of the population at large. ${ }^{1}$ This suggestion has originated from studies of death certificates, community cohorts, and death rates of institutionalised patients. ${ }^{2-7}$ The cause of this excess mortality remains uncertain, although deaths due to neoplasms may be increased in patients with epilepsy, in addition to deaths directly related to the epilepsy.

The Chalfont Centre for Epilepsy is a tertiary referral centre for epilepsy and it provides assessment, rehabilitation and a long term residential care centre for people with severe chronic epilepsy. We have investigated the causes of death in all patients in the long term residential care unit at the centre over an 11 year period, to ascertain mortality rates for specific conditions.

\section{Methods}

Death certificates and necropsy reports relating to all deaths of patients in the long term residential care unit at the Chalfont Centre for Epilepsy between 1 January 1980 and 31 December 1990 were obtained. Surveys of the numbers of residents, with demographic details for the period were used together with tables of national mortality rates from the Office of Population Census and Surveys (OPCS) to estimate the expected mortality in this group and to calculate standardised mortality ratios (SMR) ${ }^{8-10}$ where possible. SMR is the ratio of deaths observed in a group to the numbers of deaths that would be expected to have occurred during a follow up period if the group in question had experienced the same age- and sex-specific death rates as the general population.

Sudden unexpected death was defined as a non-traumatic unwitnessed death occurring in a patient who had been previously healthy (apart from epilepsy), or suffering from a disease which would not ordinarily be expected to produce immediate or sudden death and for whom no cause was found after necropsy examination. Death occurring during or immediately after a witnessed seizure was classified separately as a seizure related death, even in those cases where no cause for death was found after a necropsy examination.

No correlation between drug treatment and cause of death was attempted. All patients in the study were on antiepileptic therapy, the most commonly prescribed over this period were carbamazepine, phenytoin, barbiturates and sodium valproate- often in a combination of 2 or 3. In the early years of the study, however, most patients were taking barbiturates and phenytoin.

\section{Results}

A total of 3392 patient-years were surveyed. The mean age for the residents surveyed was 52 years (range 18-91 years); the male:female ratio in the group was $2: 1$.

Over the study period there were 113 deaths, which compared with 58.3 expected deaths in this population $(S M R=1 \cdot 9 ; 95 \%$ CI $1 \cdot 6-2 \cdot 3$; $p<0.01)$. The causes of death fell into 6 main categories: neoplasms $(n=29 ; 26 \%)$, bronchopneumonia $(n=28 ; 25 \%)$, circulatory diseases $(n=27 ; 24 \%)$, seizure-related death $(n=13 ; 12 \%)$, SUD $(n=7 ; 6 \%)$ and accidents $(n=3 ; 3 \%)$. Other causes accounted for the remaining 6 deaths. The mean age at death for all patients and for individual causes is given in table 1 . Necropsy reports were available for $88(78 \%)$ patients. All patients in the seizure-related and sudden unexpected death 
Table 1 Cause and age at death (years)

\begin{tabular}{lll}
\hline & Mean & Range \\
\hline Bronchopneumonia & 69 & $(46-89)$ \\
Cancer & 64 & $(38-91)$ \\
Circulatory Disease & 72 & $(35-91)$ \\
Seizure related & 46 & $(23-69)$ \\
Sudden & 38 & $(24-56)$ \\
Overall & 64 & $(23-91)$ \\
\hline
\end{tabular}

group had necropsy examination; the great majority of the patients who did not have necropsy were in the elderly age-group with bronchopneumonia or circulatory diseases.

There was an excess mortality due to cancer in this group (SMR $=2.0 ; 95 \%$ CI $1 \cdot 3-2 \cdot 9$; $p<0.01$ ). This surplus originated mainly from increased rates of tumours of the lung, pancreas hepatobiliary system and lymphatic/haemopoeitic tissue. In addition, there was a small contribution from tumours at a variety of other sites. Table 2 shows the SMRs for the different neoplasms in this population. There was only one CNS neoplasm amongst this group.

Twenty seven patients died from disorders of the circulation (ischaemic heart disease and cerebrovascular disease)-(SMR $0.77 \quad \mathrm{CI}$ $0 \cdot 5-1 \cdot 1)$. Of these, 6 patients had cerebrovascular accidents. Sudden unexpected death where no cardiac lesion was found at necropsy examination were not included in this group.

Bronchopneumonia was common and terminal in debilitated patients, accounting for 28 deaths. Such deaths tended to occur in an elderly age group (table 1) as did those with circulatory disorders.

Seizure related death accounted for 13 $(12 \%)$ deaths, and tended to occur in a younger age-group (table 1). One death resulted from status epilepticus and the remaining 12 were related immediately to a seizure. In half of these cases there was evidence for either aspiration or asphyxiation, and in the other half no structural cause could be found at necropsy to account for the death.

Seven deaths were sudden and unexplained; these were again in a younger group (mean age 38 ) and most of these were male $(n=5)$. The most common scenario was a patient found dead in bed with no evidence of a seizure having occurred.
Three of the deaths were due to accidents; drowning in two cases and 1 case of acute high cervical cord compression in a fall following a seizure. Two patients died of renal failure. Pericarditis, septicaemia, gangrene and pulmonary embolism accounted for one death each.

\section{Discussion}

It is generally accepted that there is an increase in mortality rates amongst patients with epilepsy. Although in the past it had been predicted that adequate seizure control with antiepileptic drugs might lead to a normal life expectancy for epilepsy patients, this seems not to be the case, except for those with absence seizures or idiopathic complex partial seizures. $^{2}$ Overall, a SMR of 1.4 to 2.9 has been found for patients with seizure disorders ${ }^{126}$ This finding is confirmed in this group of patients with severe epilepsy. The patients in this retrospective study, however, represent a highly selected group and are by no means representative of the generality of patients with epilepsy. Long term residents at the Chalfont Centre have severe chronic epilepsy which has led to them being unable to live in the open community. Patients with rapidly progressive or neoplastic causes for epilepsy are not admitted to the long term care facilities, and such cases will be excluded. The highly selected nature of these patients must be borne in mind therefore in the interpretation of results.

An overall excess mortality from neoplasms was seen in this group and this has also been noted by previous investigators ${ }^{1611}$ although not by all. ${ }^{41213}$ Hauser and Hesdorffer in their authoritative review of the subject estimated an overall odds ratio for tumours as $1 \cdot 8,{ }^{1}$ although this figure includes CNS malignancies, which were not seen in this study, apart from one case of fibrosarcoma. A previous study of Chalfont Centre patients, both residents and ex-patients in the community, found an SMR of 1.4 for non-CNS tumours, without, however, a significant increase in tumours of any one site. ${ }^{6}$ This study, based on death certificates only, had been specifically investigating increases in cancer mortality as there had been suggestions that regular anti-

Table 2 Causes of death

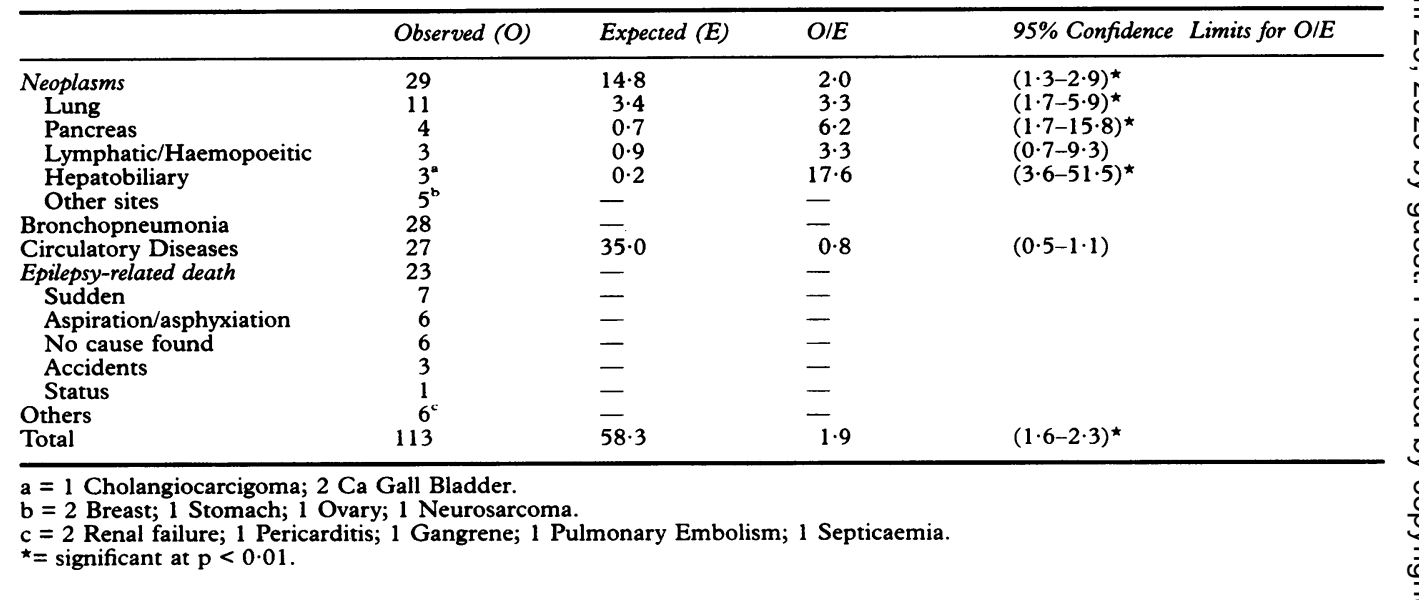


epileptic drug-intake might increase the risk of cancer in patients with epilepsy. ${ }^{14}$ The population in this previous study differs slightly from ours in that all our patients were long term residents and the drug exposure may have been dissimilar. In addition, as our patients were kept under lifetime medical supervision and usually subjected to post mortem examination, it is possible that more tumours were diagnosed, either before or after death, than would have been the case if they were in the community.

This study shows a significant increase in lung neoplasms amongst the patients, with an SMR of 3.3 although we feel that this finding should be interpreted cautiously as we have not taken into consideration the possible contribution of exposure to cigarettes. Currently, for instance, $19 \%$ of the long term residents of the centre are smokers. A similar increase in lung cancer incidence amongst patients with epilepsy has previously been noted with an odds ratio of $2 \cdot 7^{11}$ In the latter study the increase was restricted, however, to the period initially after diagnosis which would not be consistent with a causative role for antiepileptic drugs. A study of barbiturate-users in San Francisco did, however, demonstrate an increased SMR for lung cancer of $1 \cdot 7 .^{15}$ There was no relationship, however, between lung cancer and duration or intensity of use of barbiturates. Friedman suggested that induction of microsomal enzymes, notably aryl-hydrocarbon hydroxylase, may convert potential carcinogens to active forms. ${ }^{15}$

Interest in any potential increase in incidence of hepatobiliary tumours prompted the previous study at Chalfont. ${ }^{6}$ Hepatic tumours, for example, may be induced in rats by phenobarbitone. ${ }^{14}$ However, that study found no excess of hepatobiliary tumours, and no other study has demonstrated one. Our set of patients included two that died due to carcinoma of the gall bladder and one due to a cholangiocarcinoma (SMR 17.6), but no hepatic tumours. This is a significant increase over expected cases in this population. We have also found an excessive rate of pancreatic cancer in our patients $(S M R=6 \cdot 15)$. High rates of pancreatic cancer have also been noted by Friedman in his study of barbiturate users but not in studies of epilepsy. ${ }^{15}$ These findings could be artefacts of small number, and a study of the prevalence of patients with epilepsy amongst those with tumours of the biliary tree and pancreas is warranted to clarify this issue.

There is some evidence from studies of lymphoma patients that exposure to phenytoin may be a risk factor with an estimated increased risk of $2 \cdot 3 .^{16} \mathrm{~A}$ study of cancer incidence amongst patients with seizures found an odds ratio for lymphoma of $2 \cdot 9$, although the confidence limits were wide for this estimate $(0 \cdot 97-5)$. Friedman's study of phenobarbitone users also found an excess of lymphoma cases, ${ }^{15}$ and most interest has naturally focused on the known association between phenytoin and lymphadenopathy. Of our patients, two had lymphomas and one chronic lymphatic leukaemia; the increase observed in these conditions was not significant.

The high incidence of deaths through bronchopneumonia reflects a common terminal cause in an elderly, debilitated population. In other studies of institutionalised patients, approximately $25 \%$ die a respiratory death, while in studies of patients in the community, this proportion is only about $5 \% .^{25}$

Of great interest is the number of deaths in our group that could be directly attributed or associated with epilepsy. Epilepsy itself as a cause of death has been subdivided into different categories: status epilepticus, seizurerelated deaths, sudden unexpected death, and accidents. In this study death due to a seizure was used as a cause of death when the patient died during or after an attack, and no other explanation could be found at necropsy. When people died in this way without any witness they were categorised in the sudden unexpected death group rather than in the group of seizure-related death. This distinction may be arbitrary, but we nevertheless felt it was appropriate. Some clinical series of patients with epilepsy have reported proportionate rates of death of around $10 \%$ for status epilepticus $^{51017}$ and the previous Chalfont study reported that $11 \%$ of the patients died of status epilepticus. ${ }^{6}$ Only one death in this group of patients with severe epilepsy was attributed to status epilepticus. This may reflect changing practices in the completion of death certificates over time, but most likely reflects and vindicates the policy presently in practice at the Chalfont Centre for aggressive early treatment of serial or prolonged seizures, that is, a prestatus phase, thus avoiding progression to full blown status epilepticus. Twelve patients died in the immediate aftermath of a seizure and in half of these no cause for death was found at necropsy. Had their final seizure not been witnessed they would have been included in the sudden unexpected death group. This latter group accounted for 7 deaths in the period. Sudden unexpected death is said to be common in clinical series of patients with severe epilepsy but much rarer in studies in the community and this issue is still open to discussion. ${ }^{1}$ This concept in epilepsy, however, is not new and was recognised in 1910, when it was reported that $19 \%$ of 592 deaths in a centre for epilepsy were due to sudden unexpected death defined as in our study. ${ }^{18}$ An annual mortality rate of one sudden unexpected death for every 370 people with epilepsy in the community has been suggested by one study ${ }^{19}$ and our figure of $1: 480$ is close to this. If death after a seizure with no other cause is included, this figure would be 1:260 per year. A variety of explanations for sudden unexpected death in epilepsy have been proposed over the years. These have included deleterious action of AEDs, autonomic effects of a seizure affecting the heart, cardiac arrythmias, and the release of endogenous CNS opioids causing respiratory depression but a causal role has not been established for any of these. 
Many previous studies have reported higher rates of accidental death than were observed in this study. ${ }^{3-51720}$ Two patients drowned, one in a pond and the other in a bath; and one patient died from a high cervical cord contusion following a seizure. We feel that this low accident rate is due to the sheltered living environment, and may not apply to people living more independently in the community.

In summary, this study of severe long-term institutionalised patients with epilepsy seems to confirm an increase in total mortality and has suggested an increase in tumour deaths, notably of the pancreas and of the hepatobiliary systems. The cause for this is unclear, although long-term drug treatment may be relevant. Deaths directly related to epilepsy and to sudden unexpected death are also increased. Larger, prospective studies of patients in the general population are warranted.

The Epilepsy Research Group is supported by the National Society for Epilepsy, The Brain Research Trust and Action Research.

1 Hauser WA, Hesdorffer DC. Mortality. Epilepsy: frequency, causes and consequences Maryland: Epilepsy Foundation of America, 1990: 297-326.

2 Annegers JF, Hauser WA, Shirts SB. Heart disease mortality and morbidity in epilepsy. Epilepsia 1984;25:699-704.

3 Hauser WA, Annegers VF, Elveback F. Mortality in patients with Epilepsy. Epilepsia 1980;21:399-412.

4 Zielinski JJ. Epilepsy and mortality rate and cause of death. Epilepsia 1974;15:191-201.

5 Chandra V, Barucha NE, Schoenberg BS, Feskovich D. Chandra V, Barucha NE, Schoenberg BS, Feskovich D.
National Mortality data for deaths due to and deaths related to epilepsy. In: Porter R, et al, eds. Advances in epileptology vol 15. New York: Raven Press, 1984: 531-4.

6 White SV, McLean AE, Howland C. Anticonvulsant drugs and cancer. Lancet 1979;ii:458-60.

7 Hauser WA, Kurland LT. Epidemiology of epilepsy in Rochester, Minnesota. Epilepsia 1975;16:1-66.

8 Office of Population Censuses and Surveys Mortality Statistics: Causes; Tables DHS \& DHZ 1980-1990. London: HM Stationery Office.

9 Bailer JC, Ederer F. Significance factors for the ratio of a poisson variable to its expectation. Biometrics 1964;12: poisson $639-41$.

10 Morris JA, Gardner MJ. Statistics in medicine. BMf 1988;296: 1313-16

11 Shirts SB, Annegers VF, Hauser WA, Kurland LT. Cancer incidence in a cohort of patients with seizure disorders. $f$ Nat Cancer Inst 1986;77:83-87.

12 Iivanaijen $M$, Lehtinen $U$. Causes of death in institutionalised epileptics. Epilepsia 1979;20:485-92.

3 Clemmensen J. Are anticonvulsants oncogenic? Lancet 1974;i:705-7.

14 International Agency for Research on Cancer. Monographs on the evaluation of carcinogenic risk to man; vol. 13, 1977.

15 Friedman GD. Barbiturates and lung cancer in humans. $\mathcal{f}$ Nat Cancer Inst 1981;67:291-5.

16 Anthony UU. Malignant lymphoma associated with hydantoin drugs. Arch Neurol 1965;22:450-4.

$17 \mathrm{Krohn} \mathrm{W.} \mathrm{Causes} \mathrm{of} \mathrm{death} \mathrm{among} \mathrm{epileptics.} \mathrm{Epilepsia}$ 1963;4:315-21.

18 Munson M. Death in epilepsy. Medical Record 1910; 77:68-72.

19 Leestma JE, Walczak T, Hughes UR, Kalelkar MB, Teas SS. A prospective study of SUD in epilepsy. Ann Neurol 1989:26:195-203.

20 Hashimoto K, FukushimaY, Saito F, Wada K. Mortality and cause of death in patients with epilepsy over 16 years of age. Fap f Psychiat Neurol 1989:43:546-7. 